\title{
Mineral Reserves under Price Uncertainty
}

Evatt, G.W. and Soltan, M.O. and Johnson, P.V. 2012

Manchester Institute for Mathematical Sciences

School of Mathematics

The University of Manchester

\footnotetext{
Reports available from: http://eprints.maths.manchester.ac.uk/

And by contacting: The MIMS Secretary

School of Mathematics

The University of Manchester

Manchester, M13 9PL, UK
} 


\title{
Mineral Reserves under Price Uncertainty
}

\author{
G.W. Evatt, ${ }^{*}$ M. Soltan \& P. V. Johnson \\ School of Mathematics, University of Manchester, Manchester, M13 9PL, UK
}

\begin{abstract}
National reporting organizations and regulatory bodies for the minerals and mining sector are requiring publicly reported OreReserve estimates to take account of uncertainties. Whilst methodologies that account for physical uncertainty appear relatively well developed, methodologies which can take account of economic uncertainty appear much less so. To counter this shortfall, we present an efficient and general methodology which can quantify the effect of price uncertainty within reserve estimates, providing both the expected reserve size and the associated distribution (box whisker plot). This statistical information can be used by interested parties to understand precisely where the reserve risks lie, which we highlight in a worked example.
\end{abstract}

Keywords: reserves, uncertainty, price, natural resource, real options, mining

\section{Introduction}

Publicly reported mineral reserve estimates for mining projects contain numerous types of uncertainty and must be made to regulatory standards (Weatherstone, 2008). Such reserve estimates inform not only corporate investors (Morley et al., 1999), but also environmental debate (Mudd, 2007), local communities (Otto, 2010) and policy decisions (Hodges, 1995). The underlying uncertainties are clearly forms of Modifying Factors, which are stated influences upon the determination of reserve estimates (CRISCO, 2011). These uncertainties come from diverse sources, such as geological estimates and unpredictability in the future commodity price (Dimitrakopoulos and Sabour, 2007). However, whilst reserve estimation methodologies which account for physical uncertainty appear relatively well developed (Stoker, 2011; Wang et al., 2010; Yamamoto, 1999), defensible methodologies for including economic uncertainty ${ }^{1}$ into reserve estimates appear much less so. This apparent methodological absence is not due to a lack of necessity, as economic uncertainty is one of the most dominant issues facing modern mining operations (Deloitte, 2011) and is a "critical" component in the making of reserve estimates (Dominy et al., 2002). This point is further evidenced by a ten year review of North American gold mining operations, which showed that the single largest cause for mine closures was a decline in gold price (Moel and Tufano, 2002).

Specific regulators and national reporting organizations produce guidelines that provide minimum standards for mineral reserve reports. In so far as they define a 'reserve' to be the portion of the total orebody that is economic to extract, it is clear

\footnotetext{
*Tel: +44161305 5830; Fax: +44161275 5819

Email address: geoffrey.evatt@manchester.ac.uk ()

${ }^{1}$ By 'economic uncertainty', we mean financial uncertainties (such as those found in commodity prices, interest rates and foreign exchange rates) that continuously impact upon a mines extraction status up until expiry.
}

that variations in price alter reserve projections (Ray, 1984). As such, regulatory guidelines for reserve estimates should (strictly) take account of potential economic variability over the whole lifetime of the project (Ludwig et al., 1993). Such guidelines include the Securities and Exchanges Commission (SEC) Industry Guide 7, the United Nations Framework for Classification for Solid Fuels and Mineral Reserves, the Committee for Mineral Reserves International Reporting Standards (CRISCO) International Reporting Template, and the Australian Stock Exchanges' JORC code. All of these guidelines make similar distinctions between the reporting of 'Probable' and 'Proven' reserves (Weatherstone, 2008), which clearly reflect degrees of certainty around the reserve estimates. Further, the JORC code makes it mandatory for reserve reports to convey a level of "confidence in the estimate" (Stoker, 2011), implying that additional statistical measures such as quartiles or error bars, must also be calculated and published in the reserve reports (Dominy et al., 2002). Yet as previously mentioned, methodologies for taking account of economic uncertainty in reserve estimates appear sparse (Afshin et al., 2009) ${ }^{2}$, implying a shortfall exists between what existing technologies can deliver and what significant regulators require.

Concurrent to the development in reporting requirements, advances have been made in methodologies that produce reserve valuations which include price uncertainty over the lifetime of the project (Abdel Sabour and Dimitrakopoulos, 2011; Grobler et al., 2011; Chen and Forsyth, 2007; Thompson et al., 2004; Slade, 2001). These methodologies rely upon finding the additional value that a responsive or adaptive operating regime would provide, when compared to a deterministic one, e.g. the cost saving effect that abandoning a mining operation has if the commodity price falls sufficiently far. Equations which

\footnotetext{
${ }^{2}$ Whilst the methodological shortfall was noted by these authors, their motivational study only considered the effect of uncertainty in the pre-extraction planning stages of the mine.
} 
determine the mine valuation are generally constructed via financially-based hedging arguments (Brennan and Schwartz, 1985), but they can also be calculated via the Feynman-Kac equation (Øksendal, 2003). In the context of this discussion, the Feynman-Kac equation has a distinct advantage over the hedging arguments, and that is its generality: it considers a broader class of expectation than valuation alone. In relation to mining, it was shown that such expectations can include the probability of project completion and the expected lifetime of the mine (Evatt et al., 2011), which are quantities that are of use to mine operators and policy makers. However in all of these studies, expectations relating to the reserve size were not considered, despite its importance to regulators, company investors and local communities.

We propose that further expansion of the work presented in Evatt et al. (2011), can provide a methodology that determines a defensible expected reserve size and distribution of risk, which takes account of price uncertainty. After all, since falls in commodity prices can cause the unexpected early closure of mines (Moel and Tufano, 2002), it is important that a risk-based methodology is developed so as to quantify the effect of such decisions upon reserve size estimates. To help achieve this, we make our own intuitive distinction between resource and reserve to be: The resource is an amount of saleable ore that is extractable if the current price level remains fixed, whereas the reserve is the amount that is expected to be extracted when price uncertainty is present ${ }^{3}$. Consequently, we may define the associated measure of risk as: the dimensionless ratio between the reserve size and the resource size; a high percentage ratio implies that one expects there to be a low chance of closure and thus most of the ore will be extracted, whereas a low percentage ratio implies a higher closure risk and thus a smaller quantity of ore is expected to be extracted. As such, we are comparing a deterministic quantity (resource) with an uncertain quantity (reserve), to provide an easily understandable relative measure of risk. This means that the presented methodology may be used to overlay a companies extraction schedule (which are generally calculated within a deterministic price environment (Monkhouse and Yeates, 2005)) with price uncertainty, to isolate and quantify the effect this Modifying Factor has upon the reserve estimate.

This methodology is derived in Section 2, where 2.1 explains how to calculate the quantity of ore that is expected to be extracted and 2.2 explains how to calculate the amount of ore that is likely to be extracted with a specified $X \%$ likelihood. We apply this methodology to a case-study mine in Section 3, where we investigate the results sensitivity to price uncertainty and resource size. We then discuss the merits and possible extensions of this methodology in Section 4, before concluding our work in Section 5.

\footnotetext{
${ }^{3}$ Clearly one could alter the name of these definitions without altering any of the concepts or methodology. So whilst this distinction is largely semantics, we have chosen it as it seems broadly in keeping with the spirit of the JORC code.
}

\section{Reserve Estimation Methodology}

For the sake of purpose and clarity, within this paper we focus upon capturing the effect of a single dominant economic uncertainty: price. This is because price is both a primary driver of cash flows and generally has a higher degree of uncertainty surrounding it than other economic drivers. It is also because a market price is an exogenous uncertainty which is hard for an individual mining firm to avoid or alter. That said, the modelling approach we use can be extended to include additional economic Modifying Factors, as highlighted in Section 4.

A common assumption within the study of mathematical finance, is for the price process $S_{t}$ to be described by the general form

$$
d S_{t}=a\left(S_{t}, t\right) d t+b\left(S_{t}, t\right) d B
$$

where $a$ is the price drift, $b$ is the price volatility and $B$ is a Wiener process. This assumption is also common throughout real options, and we likewise maintain it. Since this paper is concerned with a 'real-world' quantity, the drift $a$ of this process must also be a real-world drift, and not a risk-adjusted drift as can sometimes be suitable if constructing a valuation (Sarkar, 2000). As such, our modelling is free of some of the recent criticisms laid at contingent-claims valuations (such as being able to continuously hedge).

The (mathematical) state-space of this store-like ${ }^{4}$ problem is given by $\left(S_{t}, Q_{t}, t\right)$, where $Q_{t}$ is the level of ore remaining within the mine (Brennan and Schwartz, 1985; Chen and Forsyth, 2007). The reduction in $Q_{t}$ is determined by the rate of extraction $q=q\left(S_{t}, Q_{t}, t\right)$ from the mine. During a small increment of time, the amount extracted can be written as

$$
d Q_{t}=-q d t
$$

which is in the form of a (trivial) stochastic differential equation.

Within this paper, we are interested in the expected amount of ore that will be extracted from the Earth, which is clearly dependent upon whether the mine owner holds the option to close the mine down early if the underlying price drops to a pre-calculated level, denoted by $S^{*}=S^{*}\left(Q_{t}, t\right)$. By noting that equation 2 tells us how much ore will be extracted within a very small period of time, we can write the expected amount of ore that will be extracted over the whole lifetime of the mine as

$$
R=\mathbf{E}_{x}\left(\int_{0}^{t^{*}} q d t\right),
$$

where $t^{*}$ is the (possibly random) time at which extraction ceases either due to mine exhaustion or mine abandonment, and $\mathbf{E}_{x}$ is the expectation given the initial conditions $x=$ $\left(S_{0}, Q_{0}, t=0\right)$. Since $R$ is the total amount expected to be

\footnotetext{
${ }^{4}$ We refer to a 'store' because the mathematical class of this problem belongs to one in which a form of store is to be controlled, where in this instance the store is the amount of ore. Related problems can be found in water management, oil extraction and gas storage (often these problems also involve forms of refilling, which this paper does not need to consider).
} 
extracted, and clearly takes account of uncertainty, we refer to this as the reserve size.

As well as the closure decision, the cut-off grade strategy will clearly also alter the reserve estimate. Such a strategy might be calculated under a deterministic price assumption (as commonly used within the planning stages of mining (Whittle and Whittle, 2007; Monkhouse and Yeates, 2005; Osanloo and Ataei, 2003)) or under an uncertain price environment (Johnson et al., 2011). Yet whichever assumption is selected, they still both give rise to a form of abandonment surface $S^{*}\left(Q_{t}, t\right)$ and extraction rate strategy $q\left(S_{t}, Q_{t}, t\right)$. Since the presented mathematics is general to all forms of $S^{*}$ and $q$, it means that our methodology is compatible with any mines prescribed cut-off grade strategy and closure criteria.

With the expectation given by equation 3 and the processes defined by equations 1 and 2, we are in a position to employ the well-known Feynman-Kac partial differential equation (PDE) (Øksendal, 2003), which enables one to efficiently calculate the size of expectation in question. The fact that the Feyman-Kac equation is very general, means that one can use it to calculate expectations beyond just that of equation 3. Indeed, for the purposes of this paper, we must calculate two such expectations: the expected reserve size and the expected probability of project completion. This second expectation is required so as to determine the percentiles of the reserve distribution, which can be used to view the spread of reserve risk (which we choose to present in the form of a box whisker plot) and thus acts as a measure of confidence in ones reserve size estimates.

\subsection{Expected Reserve Size}

To calculate the expected reserve size given by equation 3, the Feynman-Kac formula allows us to write

$$
\begin{array}{ccc}
R=0 & \text { when } \quad t=T \\
R=0 & \text { when } \quad Q=0 \\
R=0 & \text { on } \quad S=S^{*} \\
R \rightarrow Q & \text { as } & S \rightarrow \infty .
\end{array}
$$$$
\frac{\partial R}{\partial t}-q \frac{\partial R}{\partial Q}+\frac{b^{2}}{2} \frac{\partial^{2} R}{\partial S^{2}}+a \frac{\partial R}{\partial S}+q=0
$$

The mathematical structure of the Feynman-Kac equation with resource constraints, sometimes requires particular numerical algorithms to extract robust solutions, such as that described in Chen and Forsyth (2007). This would be particularly true if one could also expand or dynamically alter the cut-off grade due to price fluctuations Johnson et al. (2011). One could alternatively use Monte-Carlo methods, provided one uses a suitably large number of simulations and time-steps.

There is a way to extract a close form approximation to the solution of this problem, which we denote by $\bar{R}$. We can achieve this by assuming a constant $q$ and $S^{*}$ (one might use averaged values of these quantities) and imposing that the price process follows a geometric Brownian motion: $a=\mu S$ and $b=\sigma S$, where $\mu$ is a constant percentage drift and $\sigma$ is a constant percentage volatility. Under these assumptions, it has been shown in Evatt et al. (2011) that an equation with identical mathematical structure to equation 4 , has an exact closed form solution given by

$$
\begin{aligned}
& \bar{R}=q T- \\
& \int_{0}^{T} \frac{q \log \left(S / S^{*}\right)(T-z)}{2 \pi \sigma^{2} z^{3}} \exp \left(-\frac{\left(\log \left(S / S^{*}\right)-\sigma^{2} \alpha z\right)^{2}}{2 \sigma^{2} z}\right) d z
\end{aligned}
$$

where $\alpha=1 / 2-\mu / \sigma^{2}$. This result could be used by practitioners who wish to more easily study effect the option to abandon has upon reserve estimates ${ }^{5}$. Calculation of this integral could be made within a spreadsheet via standard integration methods, such as Simpsons Rule.

\subsection{Reserve Distribution}

The methodology we use to determine specified percentiles of the reserve distribution, requires calculation of the probability of project completion $P$. Whilst the derivation of the probability of completion is fully detailed in Evatt et al. (2011), we replicate the resulting PDE here so as to clarify the methodology for the reader:

$$
\begin{gathered}
\frac{\partial P}{\partial t}-q \frac{\partial P}{\partial Q}+\frac{b^{2}}{2} \frac{\partial^{2} P}{\partial S^{2}}+a \frac{\partial P}{\partial S}=0 \\
P=1 \quad \text { when } \quad t=T \\
P=1 \quad \text { when } \quad Q=0 \\
P=0 \quad \text { on } \quad S=S^{*} \\
P \rightarrow 1 \quad \text { as } \quad S \rightarrow \infty
\end{gathered}
$$

Once $P$ has been calculated, one must invert this calculation to find the reserve size that gives rise to a given probability of mine completion, and then repeat this process for each required percentile. For example, if one wished to know the initial $(t=0)$ amount of ore that was $X \%$ likely to be fully extracted, $Q_{0}^{X}$, one must first calculate the probability of project completion throughout the full solution space, and then find the $Q_{0}^{X}$ whose initial probability of full extraction is $X \%$. Mathematically, one is searching for the root $\left(Q_{0}^{X}\right)$ of the inverse problem: $P^{-1}\left(S_{0}, Q_{0}^{X}, 0\right)-X \%=0$.

The fact that one needs to first calculate the probability of project completion throughout the $Q$ dimension, means the PDE approach presented here is particularly efficient, in that only two iterations of algorithms are required. Conversely, the fact that one does not initially know $Q_{0}^{X}$, means that a MonteCarlo approach would be much more time-intensive; a simulation would have to be run for each possible initial reserve size, magnifying the required number of iterations.

Under the same conditions used to derive the closed-form solution of equation 5 (geometric Brownian motion and constant $q$ and $S^{*}$ ), a closed form solution to equation 6 was presented in

${ }^{5}$ Because $q$ must be averaged for this result to hold, one cannot use this equation to determine the approximate effect of a price-varying cut-off grade strategy, if there was one. 
Evatt et al. (2011) (equation 4.11). Again, that result could be used to determine accurate approximate solutions to the spread of reserve risk in the same root-finding manner as that described above, but this time via a more simple solution methodology than might otherwise be available.

\section{Results}

We now apply our methodology to a case study open pit mine, as used in Evatt et al. (2011), which has a resource size measured in tonnes of gold. The original data was supplied by Gemcom Software International, and represents a block by block extraction schedule from a series of nested pits (calculated using the Gemcom-Whittle algorithm). Whilst the focus of this paper does not critically depend up how the extraction schedule was determined, we note that the schedule is made inclusive of stockpiling and cut-off grade considerations, under an assumption of a deterministic price. Furthermore, all prices within this paper have been normalized to a (supplied) reference level.

To provide a worked example, we must specify a more precise form of the price process. Without loss of generality, we choose the price to follow a geometric Brownian motion: $a=\mu S$ and $b=\sigma S$. To make our results easily repeatable, this example assumes a constant (normalized) abandonment price, as calculated via an approximation shown in Evatt et al. (2011) (equation 3.4). With this in mind, we specify our example parameter values to be:

$$
\begin{gathered}
\mu=0 \% \mathrm{yr}^{-1}, \quad \sigma=30 \% \mathrm{yr}^{-\frac{1}{2}} \quad \mathrm{~T}=15 \mathrm{yr}^{-1}, \\
q=1950 \text { tonnes } \mathrm{yr}^{-1}, \quad \mathrm{~S}^{*}=0.51 .
\end{gathered}
$$

Using the above parameter values, we now solve equations 4 and 6 to determine the associated reserve risk. The fact that we are using constant (averaged) values for $q$ and $S^{*}$, means that it would be possible for users to replicate our results either through numerical methods or their closed-form solutions. The results are shown in Figure 1 where the continuous bold line is the behavior of the expected reserve size (as a fraction of the resource size) as one varies the initial ore price. To provide information regarding the spread of reserve risk, the associated box whisker plots are also plotted, where each point of the box denotes the fraction of ore that is $X \%$ likely to be extracted. Within the box-whisker plot, the ends of the whiskers represent the $X=5 \%$ and $X=95 \%$ levels, the ends of the boxes are the $X=25 \%$ and $X=75 \%$ levels and the bold line contained within the box is the median value $(X=50 \%)$.

Figure 1 also demonstrates that the distribution of the reserve size for a given ore price is non-symmetric, where the skew depends upon the initial conditions of the system, and the inter-quartile range can be in excess of $50 \%$. Also, the spread of the distribution decreases for higher prices, reflecting the associated lower probability of project abandonment. This quantitative picture for the spread of reserve risk is complemented by the empirical study of Moel and Tufano (2002), which demonstrated how lower gold prices can significantly affect the amount of ore recovered from a mine.
To highlight the sensitivity of the reserve size distribution to price volatility, Figure 2 shows two results that use the same parameters as before, but with the price volatility decreased to $\sigma=20 \%$ (Figure 2 left) and increased to $\sigma=40 \%$ (Figure 2 right). Since increased volatility infers increased uncertainty of the future price level, one would expect a larger likelihood of mine abandonment, and thus a smaller expected reserve size; this is confirmed by the results.

A natural point to investigate next, is to see how sensitive the reserve distribution to the size of the resource. To make a fair comparison, we vary the previous examples resource size by \pm $33 \%{ }^{6}$. The reserve size distribution for the larger mine is shown in Figure 3 right, and the reserve size distribution for the smaller mine is shown in Figure 3 left. Due to the increased potential lifetime of the larger mine, it is more likely that the price might decline far enough so as to warrant abandoning the operation, and therefore the larger mine carries with it a lower expected percentage of ore to be extracted (although, in absolute terms the expected quantity is obviously larger).

\section{Discussion}

This methodology is very well suited to take account of the core (price-sensitive) decisions relating to the operational state of the mine, such as abandoning, mothballing or expansion (Dixit and Pindyck, 1994). Additional decisions are obviously available to the operator, and could often be accounted for by this methodology. However, it must be remembered that this methodology concerns decisions which significantly contribute to the reserve estimate specifically via economic variations, rather than geophysical ones (for which other approaches are available Rendu (2002)). In addition, one can side-step certain aspects of mine planning (such as the option to switch the location of extraction within a given mine), by simply using an averaged ore-grade value (Wang et al., 2010).

The fact that the expected reserve size is both not intensive to calculate and could be obtained via appropriate Monte-Carlo methods, means that this quantity could be used by practitioners without a background in mathematics. To this effect, the closed form solution given by equation 5 should help practitioners even further. The calculation for the spread of reserve risk is slightly more involved, but as the results clearly demonstrate, there is a lot of valuable information contained within the whisker plots which can be helpful in a extraction projects risk assessment. In addition, calculating the distribution of the reserve risk explicitly answers calls made by Dominy et al. (2002), for further statistical information to be provided within publicly reported reserve estimates. As such, the (relatively modest) additional involvement required to calculate the box whisker plots, might well be worth the effort.

\footnotetext{
${ }^{6} \mathrm{We}$ are able to conduct this experiment in a transparent like-for-like manner, by assuming the ore grade within the earth is homogeneous, and thus most variables and mine planning considerations can be treated as identical in each situation (with particular note to the fixed $S^{*}$ ). Practically, this is achieved by changing the potential operating lifetime of the mine to $T=10$ and $T=20$ years) and maintaining all other parameter values as fixed within 7
} 




Figure 1: The expected reserve size and associated box whisker plots, as a proportion of the resource size. Within this paper, we are defining the resource to be the amount of saleable ore that is extractable if the current price level remains fixed, whereas the reserve is the amount that is expected to be extracted when price uncertainty is present
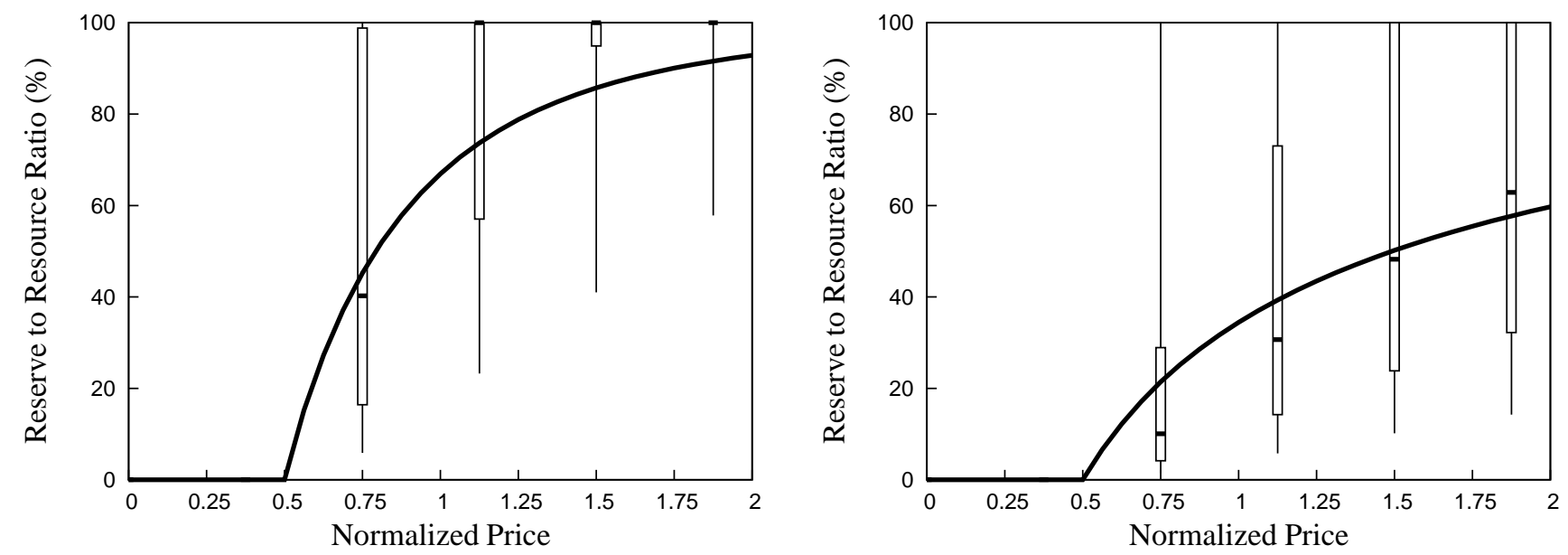

Figure 2: The expected reserve size and associated box whisker plots as a proportion of the resource size. These examples have an underlying $20 \%$ price volatility (left) and a $40 \%$ price volatility (right). Within this paper, we are defining the resource to be the amount of saleable ore that is extractable if the current price level remains fixed, whereas the reserve is the amount that is expected to be extracted when price uncertainty is present

Global mining operations will continue to have economic uncertainty as a significant consideration (Deloitte, 2011; Riseborough, 2011; Swanepoel, 2010) and improvements for quantifying the associated risks will continue to be sought by regulators (SEC, 2011). It is therefore clear that a variety of uncertainties (Modifying Factors) should be considered within a reserve estimate. We have already noted that ore-grade uncertainty is better utilized in reserve estimates than economic uncertainty. So beyond our explicit consideration of price uncertainty, one might wish to also consider including operating cost uncertainty (Dehghani and Ataee-pour, 2012), or interest rate and foreign exchange risk (provided they can be written in the form of a stochastic process, which these mentioned uncertainties generally can). Fortunately, the modelling approach we have used (the Feynman-Kac equation), is designed so as to include multiple uncertainties, and thus equation 4 and 6 can easily be extended to higher dimensions. Some care must be taken though, as the addition of further uncertain terms rapidly causes the 

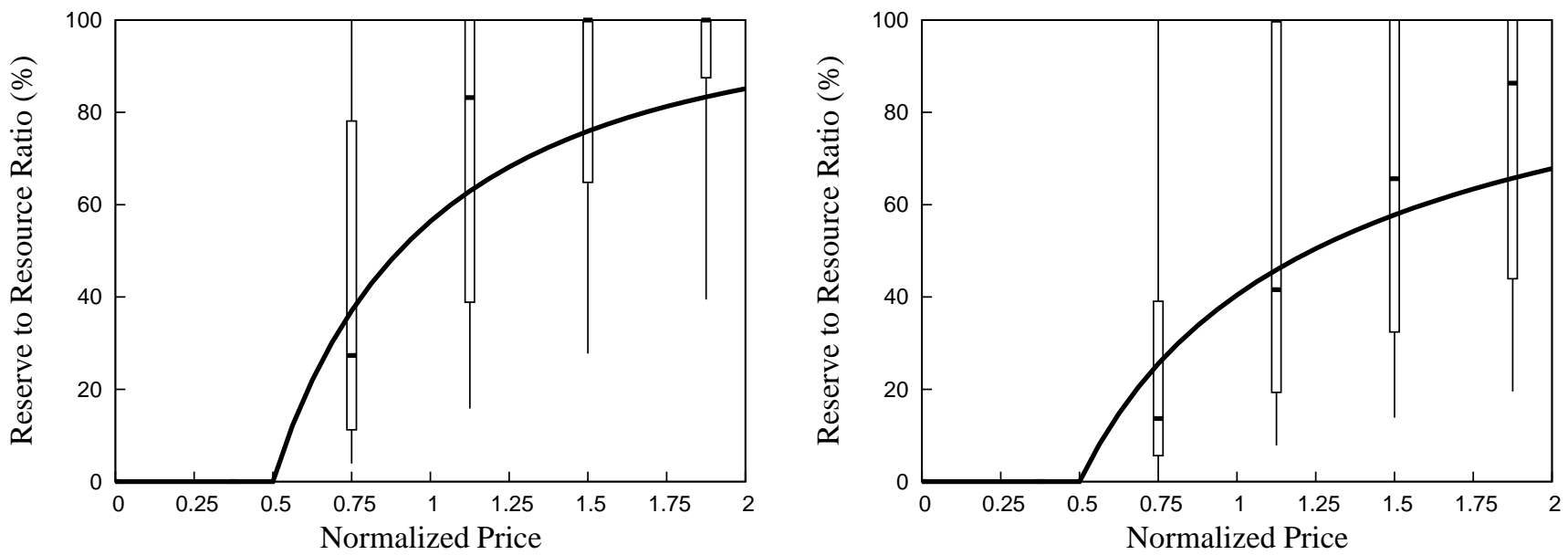

Figure 3: The expected reserve size and associated box whisker plots as a proportion of the resource size. These example have a resource size that is $33 \%$ smaller (left) and 33\% larger (right), than the mine used in Figure 1. This is able to be achieved within our experiments, by simply varying the parameter $T$. Within this paper, we are defining the resource to be the amount of saleable ore that is extractable if the current price level remains fixed, whereas the reserve is the amount that is expected to be extracted when price uncertainty is present

problem to be intractable to solve in real time ${ }^{7}$. However, the fact that within Financial Option modelling, one generally sees the price uncertainty to capture the vast majority of added information, and including additional uncertainty tends to have rapidly decreasing effects upon the results, this should help alleviate some concerns in regards accuracy of this more general modelling approach.

\section{Conclusions}

The consequence of a mine owner conducting an operating regime that responds to price fluctuations, is that a risk surrounds the amount one hopes to extract prior to closure (either through cut-off grade changes or abandonment due to unfavourable prices). This paper has presented a methodology that enables this Modifying Factor of price uncertainty to be included within such a reserve estimate. By making a suitable definition of resource and reserve, we have been able to derive an understandable measure of reserve risk, that directly reflects the possible effect of changing operational status. It is hoped that this measure of risk will be useful in the reporting of mineral resources and reserves, where significant regulators are increasingly wanting company statements to take account of uncertainty.

The methodology can also be used to provide practitioners with a measure of confidence in their estimate, which is also being called for within mineral reporting codes (Stoker, 2011). Furthermore, the fact that our methodology is founded in well-understood and transparent science, and appears general to all manner of mining operations (open pit, underground, dynamic/static cut-off grade, multi-ore mines etc), should act in its favour. That said, there is clearly some barrier to entry in the implementation of such mathematical technology; it is hoped

\footnotetext{
${ }^{7}$ As a crude rule of thumb for problems such as these, it would currently be difficult to consider more than three stochastic uncertainties
}

that the approximation to the expected reserve size, equation 5 , will help mitigate some of this potential difficulty, since it may be calculated using using basic numerical skills upon a standard spreadsheet package. Besides, despite new regulatory requirements occasionally being burdensome for some organizations, it is suggested that mining companies will welcome new methodologies which can contribute to improvements in the reserve estimation process (Snowden, 2001). Consequently, it is hoped that the methodology presented here, is a viable way for helping bridge a gap between industrial practice and regulatory standards for mineral reserve reporting.

\section{Acknowledgements}

Thanks to Gemcom Software International for their continuing advice regarding current mining industry practice, Jerry Huke for insightful comments, and the Engineering and Physical Sciences Research Council, UK, for funding via the CICADA grant.

\section{References}

Abdel Sabour, S., Dimitrakopoulos, R., 2011. Incorporating geological and market uncertainties and operational flexibility into open pit mine design. Journal of Mining Science 47, 191-201.

Afshin, D. A., Osanloo, M., Shirazi, M. A., 2009. Reserve estimation of an open pit mine under price uncertainty by real option approach. Mining Science and Technology (China) 19 (6), $709-717$.

Brennan, M. J., Schwartz, E., 1985. Evaluating natural resource investments. The Journal of Business 58 (2), 135-157.

Chen, Z., Forsyth, P., December 2007. A semi-lagrangian approach for natural gas storage valuation and optimal operation. SIAM J. Sci. Comput. 30, 339368.

CRISCO, 2011. Committee for mineral reserves international reporting standards, standard definitions. www.crisco.com December.

Dehghani, H., Ataee-pour, M., 2012. Determination of the effect of operating cost uncertainty on mining project evaluation. Resources Policy (0), - .

Deloitte, 2011. Tracking the trends 2011. The top 10 issues mining companies will face in the coming year. 
Dimitrakopoulos, R. G., Sabour, S. A. A., 2007. Evaluating mine plans under uncertainty: Can the real options make a difference? Resources Policy 32 (3), $116-125$.

Dixit, A., Pindyck, R., 1994. Investment under Uncertainty. Princeton University Press.

Dominy, S. C., Noppe, M. A., Annels, A. E., 2002. Errors and uncertainty in mineral resource and ore reserve estimation: The importance of getting it right. Exploration and Mining Geology 11 (1-4), 77-98.

Evatt, G. W., Johnson, P., Duck, P., Howell, S., Moriarty, J., 2011. The expected lifetime of an extraction project. Proceedings of the Royal Society A: Mathematical, Physical and Engineering Sciences 467, 244-263.

Grobler, F., Elkington, T., Rendu, J.-M., 2011. Robust decision making application to mine planning under price uncertainty. Proceedings of the 35th APCOM Symposium, 371-380.

Hodges, C. A., 1995. Mineral resources, environmental issues, and land use. Science 268 (5215), 1305-1312.

Johnson, P. V., Evatt, G. W., Duck, P. W., Howell, S. D., 2011. The determination of a dynamic cut-off grade for the mining industry. In: Ao, S.-I., Gelman, L. (Eds.), Electrical Engineering and Applied Computing. Vol. 90 of Lecture Notes in Electrical Engineering. Springer Netherlands, pp. 391403.

Ludwig, D., Hilborn, R., Walters, C., 1993. Uncertainty, resource exploitation, and conservation: Lessons from history. Science 260 (5104), 17-36.

Moel, A., Tufano, P., 2002. When Are Real Options Exercised? An Empirical Study of Mine Closings. Review of Financial Studies 15 (1), 35-64.

Monkhouse, P. L., Yeates, G., 2005. Beyond naive optimisation. Orebody Modelling and Strategic Mine Planning AUSIMM Spectrum Series 14, 379-383.

Morley, C., Snowdon, V., Day, D., 1999. Financial impact of resource/reserve uncertainty. The Journal of the South African Institute of Mining and Metallurgy (Octber-December), 293 - 301.

Mudd, G., 2007. Global trends in gold mining: Towards quantifying environmental and resource sustainability. Resources Policy 32 (1-2), 42 - 56.

Øksendal, B., 2003. Stochastic differential equations: an introduction with applications. Universitext (1979). Springer.

Osanloo, M., Ataei, M., 2003. Using equivalent grade factors to find the optimum cut-off grades of multiple metal deposits. Minerals Engineering 16 (8), $771-776$.

Otto, J. M., 2010. Community development agreement: Model regulations and example guidelines. World Bank Report, 61482 1, 1-84.

Ray, G., 1984. Mineral reserves: Projected lifetimes and security of supply. Resources Policy 10 (2), 75 - 80.

Rendu, J.-M., 2002. Geostatistical simulations for risk assessment and decision making: The mining industry perspective. International Journal of Surface Mining, Reclamation and Environment 16 (2), 122-133.

Riseborough, J., 2011. Rio ceo sees china iron ore buyers shifting to shorter contracts. Bloomburg Business Week (24th October).

Sarkar, S., 2000. On the investmentuncertainty relationship in a real options model. Journal of Economic Dynamics and Control 24 (2), 219 - 225.

SEC, 2011. Sec staff to hold roundtable on measurement uncertainty in financial reporting. Securities and Exchanges Commission Press Release 2011-215.

Slade, M., 2001. Valuing managerial flexibility: An application of real-option theory to mining investments. Journal of Environmental Economics and Management 41 (2), $193-233$.

Snowden, V., 2001. Practical interpretation of mineral resource and ore reserve classification guidelines. Mineral Resource and Ore Reserve Estimation The AusIMM Guide to Good Practice (Monograph 23).

Stoker, P., 2011. Jorc and mineral resource classification systems. Proceedings of the 35th APCOM Symposium, 69-73.

Swanepoel, E., 2010. Bhp moves to short-term iron-ore contracts. Mining Weekly (30th March).

Thompson, M., Davison, M., Rasmussen, H., 2004. Valuation and optimal operation of electric power plants in competitive markets. Operations Research 52 (4), 546-562.

Wang, Q., Deng, J., Zhao, J., Liu, H., Wan, L., Yang, L., 2010. Tonnage-cutoff model and average grade-cutoff model for a single ore deposit. Ore Geology Reviews 38 (1-2), 113 - 120.

Weatherstone, N., 2008. International standards for reporting of mineral resources and reserves -status, outlook and important issues. World Mining Congress and Expo 2008, 1-10.

Whittle, G., Whittle, J., 2007. Cut-off grade optimisation. Whittle Consulting Pty Ltd, 1-5.
Yamamoto, J. K., 1999. Quantification of uncertainty in ore-reserve estimation: Applications to chapada copper deposit, state of gois, brazil. Natural Resources Research 8, 153-163. 